\title{
Effect of autocatalysis on variant selection of $\alpha$ precipitates during phase transformation in Ti-6Al-4V alloy
}

\author{
Di Qiu ${ }^{a, b, *}$, Pengyang Zhao ${ }^{b}$, Rongpei Shi ${ }^{b}$, Yunzhi Wang ${ }^{b}$, Weijie Lu ${ }^{\text {a, * }}$ \\ ${ }^{a}$ State Key Laboratory of Metal Matrix Composites, Shanghai Jiao Tong University, 800 \\ Dongchuan Road, Shanghai 200240, People's Republic of China \\ ${ }^{\mathrm{b}}$ Department of Materials Science and Engineering, The Ohio State University, 2041 College \\ Road, Columbus, OH 43210, USA \\ *Corresponding authors: amanda77@sjtu.edu.cn (D. Qiu), luweijie@sjtu.edu.cn (W. Lu)
}

\begin{abstract}
The elastic interactions among $\alpha$ precipitates and their effects on variant selection are investigated. The stress field around a semi-coherent $\alpha$ lath is first calculated using phase field microelasticity theory and then the precipitation process of $\beta \rightarrow \alpha+\beta$ in Ti- $6 \mathrm{Al}-4 \mathrm{~V}$ is simulated using a 3-D phase field model. The orientations of secondary $\alpha$ variants induced by a primary (pre-existing) $\alpha$ variant obtained from the phase field simulations are consistent with those of variants within commonly observed $\alpha$ clusters in experiments. The formation of $\alpha / \alpha$ orientation relationship of the $[11 \overline{2} 0]_{\alpha} / 60^{\circ}$-type, $[\overline{10} 553]_{\alpha} / 63.26^{\circ}$-type and $[0001]_{\alpha} / 10.53^{\circ}$-type frequently observed in experiments could be a direct consequence of autocatalysis during nucleation and growth of $\alpha$ variants. Moreover, the competition among $\alpha$ variants nucleated simultaneously around a primary $\alpha$ lath for limited number of preferred nucleation sites also results in the selection of variants possessing specific crystallographic orientation with respect to the primary $\alpha$ precipitates.
\end{abstract}

Keywords: Autocatalytic effect; Elastic interaction; Transformation texture; Phase field simulation. 


\section{Introduction}

Two-phase $\alpha / \beta$ titanium alloys have exhibited a wide range of applications, from energy industry, bio-medical implants to aerospace technology $[1,2]$ owing to their excellent and combined properties of high strength, relatively low density and good resistance to fatigue crack initiation and corrosion [3, 4]. These balanced properties are controlled by microstructure that can be systematically designed through different types of thermomechanical processing routes [5]. As the $\alpha$ phase comprises the majority of the microstructure (e.g., for Ti- $6 \mathrm{Al}-4 \mathrm{~V}$, the volume fraction of $\alpha$ phase is $\sim 85 \%$ at $800^{\circ} \mathrm{C}$ [6]) and the crystal lattice of $\alpha$ phase (h.c.p) usually presents high property anisotropy, the mechanical behavior of final two-phase $\alpha / \beta$ alloys would vary significantly depending on the morphology of $\alpha$ phase [7, 8]. Therefore, understanding the formation mechanisms of orientation and spatial distribution of $\alpha$ precipitates is of great importance for the optimization of the processing routes of the alloys [9, 10]. During the diffusional $\beta$ (b.c.c) to $\alpha$ phase transformation, the $\alpha$ precipitates keep the well-known Burgers orientation relationship (Burgers $O R$ ) with respect to the $\beta$ matrix, i.e., $\{0001\}_{\alpha} \|\{110\}_{\beta}$ and $\langle 11 \overline{2} 0\rangle_{\alpha} \|\langle 111\rangle_{\beta}$ [11]. The reduction in symmetry during this transformation suggests that twelve crystallographically equivalent orientation variants of the $\alpha$ phase could be generated from a single $\beta$ grain [12]. Experimentally, however, it is extensively observed that variant selection takes place, wherein only a subset of the twelve variants is present in the obtained microstructure [13].

Upon cooling from above $\beta$ transus, the pre-existing defects within $\beta$ phase, such as dislocations $[8,14-16]$ and grain boundaries $[5,15,17-19]$ have been reported to have significant influences on the formation of $\alpha$ texture and variant selection process during $\beta \rightarrow \alpha$ phase transformation. Moreover, during continuous cooling the primary $\alpha$ precipitates could also impact the orientations of subsequently nucleated $\alpha$ phase through the effects of autocatalysis [3] or self-accommodation [20]. For example, fine secondary $\alpha$ lath precipitation takes place in regions of $\beta$ retained near the primary $\alpha$ laths, which may be attributed to the inducement of the primary $\alpha$ laths [21]. Besides, it is reported that local stresses will promote the formation of $\alpha$ variants whose orientation is similar with that of the primary one [22]. More specifically, Humbert et al. [8] and Germain et al. [7] observed that the $c$ axes of secondary $\alpha$ and primary $\alpha$ 
grains coincide with each other. Besides, Bohemen et al. [3] found that the clusters most frequently observed are constituted by different variants either sharing a common $\langle 11 \overline{2} 0\rangle_{\alpha} \|\langle 111\rangle_{\beta}$ direcion or a common $\{0001\}_{\alpha} \|\{110\}_{\beta}$ plane. Formation of those "textured" secondary $\alpha$ in the vicinity of primary ones or the gathering of several specific $\alpha$ variants within a single cluster must be closely related to the mutual interaction among these variants but is still lacking in detailed analysis and concrete proof.

At the theoretic level, by employing the phenomenological theory of martensitic transformations [23], clusters of special misorientation may result from the accommodation of shape strains associated with three or four variants, which can minimize the strain energy [20]. Moreover, according to the micro-elasticity theory [24], one can quantify how much a single $\alpha$ variant favors or disfavors the other individual variant by calculating the average density of the so-called configuration-independent part of elastic energy [25]. However, both approaches do not take account of neither the shape of each individual variant nor the spatial arrangements of multiple precipitates, which will, however, alter significantly the internal stress field during the temporal evolution of microstructure and thus influence the subsequent precipitation behavior.

Our present work aims at investigating the elastic interaction between different variants and its correlation with the formation of frequently observed types of $\alpha / \alpha-\mathrm{OR}$. In the following sections, we first introduce the method of calculating stress field associated with an arbitrary configuration of inclusions (Section 2.1) and the phase field model for $\beta \rightarrow \alpha$ phase transformation in a two-phase $\alpha / \beta$ titanium alloy, i.e., Ti-6Al-4V (Section 2.2). Then a set of carefully designed calculations and simulations are carried out and the results are shown in Section 3 and discussed in Section 4. Finally, the major findings are summarized in Section 5.

\section{Methods}

\subsection{Calculation of stress field around an $\alpha$ plate}

In general, for an elastically homogeneous solid medium containing randomly distributed Eshelby inclusions [26], where we assume that the inclusion (precipitate) has the same elastic modulus as the matrix, the stress field $\sigma_{i j}(\mathbf{r})$ due to the transformation (matrix to inclusion) strain can be 
calculated by solving the stress equilibrium equation (subjected to the ascribed boundary conditions):

$$
\frac{\partial \sigma_{i j}(\mathbf{r})}{\partial r_{j}}=0
$$

Within the framework of small-strain approximation, the total strain field of the system is related to the displacement field $u_{i}(\mathbf{r})$ :

$$
\grave{\mathrm{o}}_{i j}(\mathbf{r})=\frac{1}{2}\left[\frac{\partial u_{i}(\mathbf{r})}{\partial r_{j}}+\frac{\partial u_{j}(\mathbf{r})}{\partial r_{i}}\right]
$$

The elastic strain $e_{i j}(\mathbf{r})$ and the total strain hold the following relationship:

$$
e_{i j}(\mathbf{r})=\grave{\mathrm{o}}_{i j}(\mathbf{r})-\varepsilon_{i j}^{T}(\mathbf{r})
$$

where $\varepsilon_{i j}^{T}(\mathbf{r})$ is the stress free transformation strain (SFTS) or eigen strain field, which is determined by the SFTS tensor of every variant $\varepsilon_{i j}^{V p}$ together with its distribution described by order parameters $\eta_{p}(\mathbf{r})$ (as to be explained in the next subsection) within the matrix, i.e., $\varepsilon_{i j}^{T}(\mathbf{r})=\sum_{p=1}^{N} \varepsilon_{i j}^{V p} \eta_{p}(\mathbf{r})$. By applying Hooke's law to Eq. (3), i.e., $\sigma_{i j}(\mathbf{r})=C_{i j k l} e_{k l}(\mathbf{r})$, it is clear that $\varepsilon_{i j}^{T}(\mathbf{r})$ serves as the input in calculating equilibrium stress $\sigma_{i j}(\mathbf{r})$. Substituting Eq. (2) and (3) together with Hooke's law into Eq. (1), we have

$$
\frac{1}{2} \frac{\partial}{\partial r_{j}} C_{i j k l}\left[\frac{\partial u_{k}(\mathbf{r})}{\partial r_{l}}+\frac{\partial u_{l}(\mathbf{r})}{\partial r_{k}}\right]=\frac{\partial}{\partial r_{j}} C_{i j k} \varepsilon_{k l}^{T}(\mathbf{r})
$$

To solve this set of second-order partial differential equations, the total strain $\grave{o}_{i j}(\mathbf{r})$ and accordingly the displacement field $u_{i}(\mathbf{r})$ are divided into an average part and a local perturbation, i.e.,

$$
\grave{o}_{i j}(\mathbf{r})=\grave{o}_{i j}+\delta \grave{o}_{i j}(\mathbf{r}), \quad u_{i}(\mathbf{r})=\bar{u}_{i}+\delta u_{i}(\mathbf{r})
$$

By substituting Eq. (5), Eq. (4) becomes

$$
\frac{\partial}{\partial r_{j}} C_{i j k l} \frac{\partial \delta u_{k}(\mathbf{r})}{\partial r_{l}}=\frac{\partial}{\partial r_{j}} C_{i j k l} \varepsilon_{k l}^{T}(\mathbf{r}) .
$$

When the medium is much larger than the inclusion, $\delta u_{k}(\mathbf{r})$ and its gradient $\frac{\partial \delta u_{k}(\mathbf{r})}{\partial r_{l}}$ vanish at the far-field such that periodic boundary conditions are satisfied and the stress could be solved by 
first solving $\delta u_{k}(\mathbf{r})$ in Eq. (6) using Fourier spectral method:

$$
\left[\delta u_{k, l}\right]_{\mathbf{k}}=n_{j} n_{l}[\Omega(\mathbf{n})]_{i k} C_{i j k l} \tilde{\varepsilon}_{i j}^{T}(\mathbf{k}),
$$

where $[\Omega(\mathbf{n})]_{i k}$ is the Green' function in reciprocal space and $[\Omega(\mathbf{n})]_{i k}^{-1}=C_{i j k} n_{j} n_{l}, \tilde{\varepsilon}_{i j}^{T}(\mathbf{k})$ is the Fourier transform of $\varepsilon_{i j}^{T}(\mathbf{r})$. Then, the total strain could be solved according to Eq. (2):

$$
\delta \grave{o}_{k l}(\mathbf{r})=\frac{1}{2}\left[n_{j} n_{l} \cdot[\Omega(\mathbf{n})]_{i k} \cdot C_{i j k} \tilde{\varepsilon}_{k l}^{T}(\mathbf{k})+n_{j} n_{k} \cdot[\Omega(\mathbf{n})]_{i l} \cdot C_{i j k l} \tilde{\varepsilon}_{i j}^{T}(\mathbf{k})\right]_{\mathbf{k} \rightarrow \mathbf{r}},
$$

where $\mathbf{k} \rightarrow \mathbf{r}$ denotes the inverse Fourier transform. With Eq. (8) and known $\varepsilon_{i j}^{T}(\mathbf{r})$, the total strain, the elastic strain and therefore the stress field could be computed by substituting Eq. (8) and Eq. (5) to Eq. (3) with the Hooke's law being applied.

\subsection{Phase field model}

By incorporating the experimental thermodynamic and kinetic database, the phase field modeling can be an effective quantitative method for simulating arbitrary microstructures in a variety of phase transformation related processes [11, 27]. Temporal evolution of the unstable microstructures results from the reduction of the total free energy, which, in our current phase field model, consists of the chemical free energy, interfacial energy, elastic strain energy and the work done by the applied external stress field [28-30].

The current three-dimensional (3-D) phase-field simulation is carried out in Ti-6Al-4V alloy [12, $31,32]$ for the variant selection process occurring in the presence of primary $\alpha$ precipitates during $\beta$ to $\alpha$ phase transformation. The evolution of microstructure is characterized by two sets of time- and spatial-dependent order parameters, i.e., conserved composition fields $\left\{X_{i}(\mathbf{r})\right\}_{i=A l, V}$ for chemical non-uniformity of $\mathrm{Al}$ and $\mathrm{V}$ (the balance is $\mathrm{Ti}$ ), and non-conserved order parameter fields $\left\{\eta_{p}(\mathbf{r})\right\}_{p=1}^{12}$ for structural non-uniformity of twelve $\alpha$ variants. Across the $\alpha / \beta$ interface, both the composition and structural order parameters vary smoothly, which is subjected to the "diffusional interface" assumption. The shapes of precipitates do not need to be predetermined and the positions of interfaces are automatically tracked during the microstructural evolution, rendering phase field as a non-boundary-tracking method. The total free energy is a functional of compositions $X_{i}(\mathbf{r})$ and order parameters $\eta_{p}(\mathbf{r})$ and in return, the corresponding 
variation of free energy define the driving forces for $X_{i}(\mathbf{r})$ and $\eta_{p}(\mathbf{r})$.

Since the phase field model associated with the precipitation process has been used in our previous works $[12,25]$, we omit here the model framework and formulation that can be found in the literature (e.g., chemical free energy $\left(E^{\text {chem }}\right)$ [33] and elastic energy $\left(E^{\mathrm{el}}\right)$ [24]). It should be noted that the anisotropy in $\alpha / \beta$ interfaces are considered in our current model by incorporating the gradient coefficients matrix according to the gradient thermodynamics [33]. For the sake of studying and discussing in the following the underlying mechanism of autocatalytic effects between $\alpha$ precipitates, we will specify the elastic energy formulation between one $\alpha$ variant characterized by a SFTS tensor $\varepsilon_{i j}^{V p}$ and the stress field induced by the primary variants $\sigma_{i j}^{\alpha_{p}}(\mathbf{r})$ (calculated according to Section 2.1), which will bring in the total free energy an additional interaction term:

$$
E_{\mathrm{int}}^{\mathrm{el}}=-\int \sigma_{i j}^{\alpha_{p}}(\mathbf{r}) \sum_{p=1}^{12} \eta_{p}(\mathbf{r}) \varepsilon_{i j}^{V p} d \mathbf{r}
$$

Following Ref. [34, 35] and our previous work [25, 31], the kinetic evolution of microstructure represented by concentration field $X_{i}(\mathbf{r})$ and order parameters $\eta_{p}(\mathbf{r})$ is governed by the following equations, respectively:

$$
\begin{gathered}
\frac{1}{V_{m}^{2}} \frac{\partial X_{k}(\mathbf{r}, t)}{\partial t}=\nabla \sum_{j=A l, V} M_{k j}\left(T, X_{i}, \eta_{p}\right) \nabla \frac{\delta E^{\mathrm{tot}}}{\delta X_{j}(\mathbf{r}, t)}+\zeta_{c}(\mathbf{r}, t) \\
\frac{\partial \eta_{p}(\mathbf{r}, t)}{\partial t}=-M_{\eta}\left\{\frac{1}{\tilde{N}} \sum_{q \neq p}\left(\frac{\delta E^{\mathrm{chem}}}{\delta \eta_{p}(\mathbf{r}, t)}-\frac{\delta E^{\mathrm{chem}}}{\delta \eta_{q}(\mathbf{r}, t)}\right)+\frac{\delta\left(E^{\mathrm{el}}+E_{\mathrm{int}}^{\mathrm{el}}\right)}{\delta \eta_{p}(\mathbf{r}, t)}\right\}+\zeta_{\eta}(\mathbf{r}, t)
\end{gathered}
$$

where $M_{k j}\left(T, X_{i}, \eta_{p}\right)$ are the chemical mobilities, $M_{\eta}$ is the kinetic coefficient related to the mobility of interfaces, $\zeta_{c}(\boldsymbol{r}, t)$ and $\zeta_{\eta}(\boldsymbol{r}, t)$ are the Langevin noise terms standing for chemical and structural fluctuations, respectively.

\section{Results}

\subsection{The stress field of primary $\alpha$ plate}

The orientation relationship between each of the twelve $\alpha$ variants and the $\beta$ matrix is exhibited 
in Table 1. In general, defects (such as dislocations and grain boundaries) within the $\beta$ matrix will provide nucleation sites for $\alpha$ precipitates, and the distribution of $\alpha$ variants will deviate from that of variants precipitate from homogeneous matrix. In the current work we focus on the effects of pre-existing $\alpha$ lath on variant selection and the development of $\alpha$ clusters of special orientations.

Table 1 The orientation relationships between $\alpha$ variants and $\beta$ phase

\begin{tabular}{cccc}
\hline variant & the orientation relationship & variant & the orientation relationship \\
\hline V1 & $(0001)_{\alpha}\left\|(101)_{\beta},[11 \overline{2} 0]_{\alpha}\right\|[\overline{1} 11]_{\beta}$ & V7 & $(0001)_{\alpha}\left\|(101)_{\beta},[11 \overline{2} 0]_{\alpha}\right\|[11 \overline{1}]_{\beta}$ \\
V2 & $(0001)_{\alpha}\left\|(\overline{101})_{\beta},[11 \overline{2} 0]_{\alpha}\right\|[111]_{\beta}$ & V8 & $(0001)_{\alpha}\left\|(\overline{101})_{\beta},[11 \overline{2} 0]_{\alpha}\right\|[1 \overline{1} 1]_{\beta}$ \\
V3 & $(0001)_{\alpha}\left\|(011)_{\beta},[11 \overline{2} 0]_{\alpha}\right\|[11 \overline{1}]_{\beta}$ & V9 & $(0001)_{\alpha}\left\|(011)_{\beta},[11 \overline{2} 0]_{\alpha}\right\|[1 \overline{1} 1]_{\beta}$ \\
V4 & $(0001)_{\alpha}\left\|(01 \overline{1})_{\beta},[11 \overline{2} 0]_{\alpha}\right\|[\overline{1} 11]_{\beta}$ & $\mathrm{V} 10$ & $(0001)_{\alpha}\left\|(01 \overline{1})_{\beta},[11 \overline{2} 0]_{\alpha}\right\|[111]_{\beta}$ \\
V5 & $(0001)_{\alpha}\left\|(1 \overline{1} 0)_{\beta},[11 \overline{2} 0]_{\alpha}\right\|[11 \overline{1}]_{\beta}$ & $\mathrm{V} 11$ & $(0001)_{\alpha}\left\|(1 \overline{1} 0)_{\beta},[11 \overline{2} 0]_{\alpha}\right\|[111]_{\beta}$ \\
V6 & $(0001)_{\alpha}\left\|(110)_{\beta},[11 \overline{2} 0]_{\alpha}\right\|[\overline{1} 11]_{\beta}$ & $\mathrm{V} 12$ & $(0001)_{\alpha}\left\|(110)_{\beta},[11 \overline{2} 0]_{\alpha}\right\|[1 \overline{1} 1]_{\beta}$ \\
\hline
\end{tabular}

The effects of pre-existing $\alpha$ lath on the development of subsequent microstructure may strongly rely on its stress field, which varies with its size, orientation and shape. As has been described in Section 2.1, the stress field of $\alpha$ precipitates could be computed by solving Eq. (8) with a given $\varepsilon_{i j}^{T}(\mathbf{r})$ as input. For a single $\alpha$ lath of variant $\mathrm{Vp}$, the $\varepsilon_{i j}^{T}(\mathbf{r})$ equals the SFTS of the $\alpha$ lath (i.e., $\left.\varepsilon_{i j}^{V p}\right)$ within the precipitate and zero outside. For example, if the $\alpha$ lath is consisted of variant V1 (see Table 1), its transformation strain $\varepsilon_{i j}^{V 1}$ in the coordinate system $x\left\|[010]_{\beta}-y\right\|[\overline{1} 01]_{\beta}-$ $z||[101]_{\beta}$ could be calculated as

$$
\varepsilon_{i j}^{V 1}=\left[\begin{array}{ccc}
-0.0830 & 0.0095 & 0 \\
0.0095 & 0.123 & 0 \\
0 & 0 & 0.035
\end{array}\right]
$$

The SFTS of Vp, i.e., $\varepsilon_{i j}^{V_{p}}(\mathrm{p}=2,3, \ldots, 12)$, could be obtained by applying symmetry operation [31] to $\varepsilon_{i j}^{V 1}$ (Eq. (12)). The microstructure and the corresponding stress field of V1 is not fully 
determined until the characters of the inclusion, such as shape and size of the $\alpha$ plate, are specified. Such characters can be easily captured in phase field model, where the shape and size are represented by order parameter $\eta_{V 1}(\mathbf{r})$. Therefore, we first set a spherical nucleus of V1 at the center of the simulation box, as shown in Fig. 1(a). It should be noted due to larger size at later stage of phase transformation, the contribution of structure ledges and misfit dislocations on the $\alpha / \beta$ interfaces to the development of $\alpha$ plate should be incorporated when calculating SFTS of V1, $\varepsilon_{i j}^{V 1}[12]$. During isothermal phase transformation, the spherical nucleus gradually grows into $\alpha$ plate due to elastic strain energy and anisotropy in interfacial energy of $\alpha / \beta$ interface. At $\tau=7$, the $\alpha$ plate has a well-defined habit plane with crystal indices of $\{\overline{11} 1311\}_{\beta}$, as indicated by the blue plane shown in Fig. 1(f). The stress field around V1 at $\tau=7$ is then calculated according to the method described in Section 2.1 and a two-dimensional (2-D) cross-section of 3-D distribution of each stress component perpendicular to $z$ axis is plotted in Fig. 2.

As can be seen in Fig. 2, the stresses are mainly concentrated around the $\alpha$ plate at the habit plane (indicated by black arrows) and edges (indicated by red arrows), and vanish when moving away from the lath. Within the interior of the lath, the $\alpha$ lath is under compression, especially for normal stress $\sigma_{33}$. Notice that the distribution of stress component differs from each other. Therefore, the primary V1 lath will interact with the other eleven variants in different intensity as the SFTS components also vary from variant to variant, which leads to the nucleation of some variants through the so-called "autocatalytic effects" but suppresses the formation of the others. To remind, the autocatalysis is a phenomenon wherein an untransformed region is driven to transform by the sufficiently high enough local stresses resulting from the previously already-transformed region. Such elastic interaction is similar to the effect of prior dislocations $[25,36-38]$ and grain boundaries [39] within the $\beta$ matrix on the variant selection process during $\alpha$ precipitation.

\subsection{Morphology of $\alpha$ variants induced by primary V1 plate}

By setting V1 at $\tau=7$ (Fig. 1(f)) as the initial microstructure, phase field simulation is then carried out to study the effects of its inherent stress field on the nucleation of other variants. As 
shown in Fig. 3, the $\alpha$ precipitates induced by V1 consist of only five $\alpha$ variants, with each one rendered by one color and specified by the arrow. Among the five secondary $\alpha$ variants, V9 and V11 spread over the habit plane of V1, whereas V4, V6 andV7 are favored at the edges. All the remaining $\alpha$ variants do not precipitate out during the whole process.

It is shown in Fig. 3(a) that while small nuclei of variants V4 and V6 arrange along two sides of the V1 lath with smaller curvature, nuclei of V7 are preferred at the edge of larger curvature. Coalescence (among same $\alpha$ variants) or impingement (among different $\alpha$ variants) can take place during further growth. As can be seen in Fig. 3(c), V9 and V11 have their habit planes nearly parallel to that of V1 plate. The morphology and position of $\alpha$ precipitates induced by V1 suggest that primary $\alpha$ plate will interact with secondary $\alpha$ precipitates consisiting of different variants in very distinctive ways, which may consequently lead to the formation of $\alpha$ clusters and phenomenon of variant selection.

\section{Discussion}

\subsection{The elastic interaction energy between primary $\alpha$ lath and secondary $\alpha$ precipitates}

During the nucleation stage the local stress accompanying with the primary $\alpha$ variants provides excess driving force or barrier for the subsequent nucleation and growth of other variants. From the view of elastic interaction between primary $\alpha$ lath and secondary $\alpha$ precipitates nucleated later on, the intensity of such interaction could be quantitatively calculated through the elastic interaction energy $E^{\mathrm{int}}(\mathbf{r}, p)$, which is the variation of $E_{\mathrm{int}}^{\mathrm{el}} \quad$ (Eq. (9)) with respect to $\varepsilon_{i j}^{V_{p}}$ :

$$
E^{\mathrm{int}}(\mathbf{r}, p)=-\sigma_{i j}^{V 1}(\mathbf{r}) \varepsilon_{i j}^{V p}
$$

where $\sigma_{i j}^{V 1}(\mathbf{r})$ is the stress field of primary $\alpha$ lath of V1, shown in Fig. 2. As is indicated by Eq. (13), the $E^{\text {int }}(\mathbf{r}, p)$ is a function of position. Locally, if $E^{\text {int }}(\mathbf{r}, p)$ is negative at $\mathbf{r}$, the primary $\alpha$ lath will promote the nucleation of $\mathrm{Vp}$ at $\mathbf{r}$ since the nucleation of $\mathrm{Vp}$ will help reduce the total free energy. The magnitude of $E^{\text {int }}(\mathbf{r}, p)$, however, dicates the degree of how much V1 favors Vp locally; Otherwise if $E^{\mathrm{int}}(\mathbf{r}, p)$ is positive, the nucleation of $\mathrm{Vp}$ will be suppressed at $\mathbf{r}$. 
Therefore, the effect of $E^{\text {int }}(\mathbf{r}, p)$ on variant selection and the formation of micro-texture is reflected in two aspects: (i) not all $\alpha$ orientation variants are preferred due to their elastic interaction with stress field of primary $\alpha$ lath and (ii) the preferred nucleation sites (positions of negative $\left.E^{\text {int }}(\mathbf{r}, p)\right)$ of favored variants vary from variant to variant as $E^{\text {int }}(\mathbf{r}, p)$ is a function of position. We plot the most negative value of interaction energy $E_{\min }^{\text {int }}(p)$ associated with each variant in Fig. 4(a) and the region of $E^{\text {int }}(\mathbf{r}, p)$ with magnitude of $E_{\min }^{\text {int }}(p) \sim 0.3 E_{\min }^{\text {int }}(p)$ is highlighted in green in Figs. 4(b)-(1), to represent the preferred nucleation sites for Vp.

As has been indicated in Fig. 4, it is apparent that all variants exhibit negative $E^{\text {int }}(\mathbf{r}, p)$ values at some specific location, which will be their preferential nucleation sites during subsequent precipitation. In terms of the position of calculated potential nucleation sites, $\alpha$ variants could be divided into three groups: the first group consisting of variants preferred at the habit plane of V1, i.e., V2, V3, V5, V8, V9 and V11, the second group consisting of only variant V7 that is favored at the edge of V1, and the third group consisting of the remaining ones, such as V4, V6, V10 and V12, which are preferred mostly in positions at the edge with a little spreading towards the habit plane. Compared with the morphology of $\alpha$ vairiants induced by V1 in Fig. 3, the positions of favored variants in Fig. 3 agree well with the negative region of $E^{\text {int }}(\mathbf{r}, p)$ in Fig. 4. For example, V4 and V6 are preferred near two sides of V1, where $E^{\text {int }}(\mathbf{r}, 4)$ and $E^{\text {int }}(\mathbf{r}, 6)$ reach the most negative (indicated by white arrows in Fig. 4(d) and (f)). Such agreement is also reached for V9 and V11 that are favored at the habit plane and V7 at the top edge of V1.

However, some of the variants may share the same nucleation sites. Under such condition, the one having more negative interaction energy will possibly be more competitive and thus occupy the nucleation sites. For those absent variants of V2, V3, V5, V8, V10 and V12, although they also possess negative region of $E^{\text {int }}(\mathbf{r}, p)$, these region are largely overlapped with those with more negative $E^{\text {int }}(\mathbf{r}, p)$. Thus, the effect of autocatalytic effect between primary $\alpha$ plate and secondary $\alpha$ precipitates is actually not solely due to the magnitude of interaction energy, since 
all variants can have negative $E_{\min }^{\text {int }}(p)$ but only a subset of them appears during precipitation, as shown in Fig. 3. Our calculation and analysis suggest that the competition between variants favored at the same position also gives rise to variant selection.

\subsection{The orientation between primary $\alpha$ plate and secondary $\alpha$ precipitates}

In general, any pair of twelve $\alpha$ variants could form $66 \alpha / \alpha$ relative orientation, which in total belong to six types of $\alpha / \alpha$ orientation relationship $(\alpha / \alpha-\mathrm{OR})$ depending on crystallographic orientation of variants [20]. Taking V1 as an example, the type of $\alpha / \alpha$-OR between Vp and V1 are listed in Table 2, where the fraction of each type $P_{\text {random }}$ in the random case (where no variant selection occurs) is also listed correspondingly. The symbol "|" denotes the $\alpha / \alpha$-OR between two variants on the left and right of it, which is described by rotation axis and rotation degrees. For example, the $\alpha / \alpha$-ORbetween V1 and V4 belongs to B type, that is, the orientation of $\mathrm{V} 1$ could be obtained by rotating $\mathrm{V} 4$ around $[11 \overline{2} 0]_{\alpha}$ axis by $60^{\circ}$, and vice versa. As indicated by Table 2, when no variant selection occurs, $\mathrm{C}$ type of $\alpha / \alpha$-OR accounts for the largest fraction of $33.3 \%$.

Table 2 Types of $\alpha / \alpha$-OR betweenV1 and other variants

\begin{tabular}{|c|c|c|}
\hline $\mathrm{V} 1 \mid \mathrm{Vp}, \mathrm{p}=1 \sim 12$ & $\alpha / \alpha$-OR-Description & $P_{\text {random }}(\%)$ \\
\hline $\mathrm{V} 1 \mid \mathrm{V} 1$ & A-I (Identity) & 8.3 \\
\hline V1|V4, V6 & $\mathrm{B}-[11 \overline{2} 0]_{\alpha} / 60^{\circ}$ & 16.6 \\
\hline V1|V3, V5, V10, V12 & $\mathrm{C}-\left[\begin{array}{llll}\overline{10} & \overline{7} & 17 & 3\end{array}\right]_{\alpha} / 60.83^{\circ}$ & 33.3 \\
\hline V1|V9, V11 & $\mathrm{D}-[\overline{10} 5553]_{\alpha} / 63.26^{\circ}$ & 16.6 \\
\hline $\mathrm{V} 1 \mid \mathrm{V} 2, \mathrm{~V} 8$ & E- $\left[\begin{array}{llll}7 & \overline{17} & 10 & 0\end{array}\right]_{\alpha} / 90^{\circ}$ & 16.6 \\
\hline V1|V7 & $\mathrm{F}-[0001]_{\alpha} / 10.53^{\circ}$ & 8.3 \\
\hline
\end{tabular}

In our study, as has been demonstrated before, if the interaction energy between primary and a secondary $\alpha$ variant is negative, the fomation of this secondary $\alpha$ variant is then encouraged. For example, as shown in Fig. 3, the orientation between primary V1 and selected variants, i.e., V4, V6, V7, V9 and V11 belong to three types of $\alpha / \alpha-O R$, including B type (V1 | V4 and V1 | V6), D type (V1 | V9 and V1 | V11) and F type (V1 | V7). Special attention should be drawn that V4, V6 and V1 share a common $\langle 11 \overline{2} 0\rangle_{\alpha} \|\langle 111\rangle_{\beta}$ direction, while V7 and V1 share a common $\{0001\}_{\alpha} \|\{110\}_{\beta}$ direction. Therefore, the existence of primary $\alpha$ lath will lead to the formation 
of some special types of $\alpha / \alpha$-OR and the corresponding fractions of each $\alpha / \alpha-\mathrm{OR}$ will deviate from values in the random case as listed in Table 2. It should also be noted that unlike other types of $\alpha / \alpha-\mathrm{OR}$, the $\mathrm{B}$ type and $\mathrm{D}$ type have a unique feature that any pair of the three variants involved in these two types (i.e., B or D) will form exactly the same $\alpha / \alpha$-OR type. Taking type B as an example, such $\alpha / \alpha-\mathrm{OR}$ exists not only at the boundaries of V1|V4 and V1|V6, but also at V4 |V6.

\subsection{Comparison between mechanisms for the formation of special $\alpha / \alpha-\mathrm{OR}$}

The preferred $\alpha / \alpha$-ORs found in the current study between primary $\alpha$ lath and induced secondary $\alpha$ variant are consistent with those commonly observed in experiments. For example, Lee et al. [17] observed $\alpha$ clusters consisting of three variants (corresponding to V1, V4 and V6 in the present work), which share the same $\langle 11 \overline{2} 0\rangle_{\alpha}||\langle 111\rangle_{\beta}$ near GB allotriomorphs and they proposed that the cluster of these three variants may further proceed and eventually develop into the basketweave structure. Besides, Bohemen et al. [3] also found that the fraction of $\alpha$ cluster constituted by variants sharing a common $\langle 11 \overline{2} 0\rangle_{\alpha} \|\langle 111\rangle_{\beta}$ is much higher than that in the random case, which in their opinion, is due to the auto-catalytic effect of primary $\alpha$ lath. In terms of their arguments, our above analysis based on elastic interaction energy calculation may provide possible mechanism for the formation of such $\alpha$ clusters. Since experimental results are always limited to 2-D observations, here we present 3-D microstructure of a cluster consisting of V1, V4 and V6 in Fig. 5(a) to illustrate the $\alpha / \alpha-\mathrm{OR}$ of these three variants. Besides, a 2-D morphology of a cross section (indicated by the partially transparent plane in Fig. 5(a)) is also shown in Fig. 5(b). These three variants belong to B type $\alpha / \alpha-\mathrm{OR}$ and the habit planes of any two of them form an inclination angle of $66.3^{\circ}$ (as will be quantified later). Our interaction energy calculation shows that the three variants of the B type have strong autocatalytic effect among themselves, which could lead to a "chain-reaction" where the nucleation and growth of one variant will induce the nucleation and growth of the other nearby (see schematics in Fig. 5(c)). The intertwine of the three variants having the shape and orientation shown in Fig. 5(a)-(b) leads to a typical triangular cluster configuration (sharing a common $[11 \overline{2} 0]_{\alpha}$ ) frequently observed in experiments. However, our current model is carried out for isothermal phase transformation, while the "chain-reaction" is more likely to occur upon continuous cooling, which will be studied in a separate paper. Besides, 
the orientation of V1 and V7 observed in Fig. 3 are consistent with those of variants within the macrozone [7, 8], where $c$ axes $\left(\|\langle 0001\rangle_{\alpha}\right)$ of $\alpha$ variants coincide with each other.

Moreover, by comparing the volume of selected variants (Fig. 3) and the interaction energy magnitude (Fig. 4(a)), we also find that although V9 and V11 have less negative $E_{\min }^{\text {int }}(\sim$ $-1700 \mathrm{~J} / \mathrm{mol})$ than those of V4/V6 $\left(E_{\min }^{\mathrm{int}} \sim-2000 \mathrm{~J} / \mathrm{mol}\right)$ and V7 ( $\left.E_{\min }^{\mathrm{int}} \sim-2200 \mathrm{~J} / \mathrm{mol}\right)$, they actually have much larger volume fraction than the others (see Fig. 3). It is not surprising if we notice that despite the interaction energy argument, the orientation of habit plane or growth path also vary from variant to variant. The inclination angle $\varphi_{h p}$ between the habit planes of secondary variant and primary V1 variant on their $\alpha / \alpha$-OR type. Due to the Burgers OR and crystal symmetry, variants form same $\alpha / \alpha-\mathrm{OR}$ with V1 will exhibit same $\varphi_{h p}$ with respect to V1. The habit plane inclination angle $\varphi_{h p}$ between variants forming different $\alpha / \alpha$-OR with V1 is shown in Fig. 6.

We find that the preferential nucleation sites of V9 and V11 are located at the habit plane of V1 according to interaction energy calculation (see Fig. 4), and the $\alpha / \alpha$-OR type of V1 | V9 or V1 | V11 happens to be D type (see Table 2), which exhibits the lowest $\varphi_{h p}$. Therefore, the stress around the primary $\alpha$ lath can be largely accommodated by the coalescence V9 and V11 at the habit plane through a relatively high growth rate. On the contrary, $\varphi_{h p}$ of the other four types of $\alpha / \alpha-\mathrm{OR}$ is much larger. Consequently, over time, extensive coalescence takes place more quickly among the nuclei of V9 or V11 than that among V4, V6 or V7. Therefore, during the nucleation stage, it is the magnitude and spatial distribution of interaction energy $E^{\mathrm{int}}(\mathbf{r}, p)$ that determine the preferred variants and their nucleation sites. During the growth, however, the habit plane inclination $\varphi_{h p}$ influences significantly the relative volume fraction of every selected variant and leads to further variant selection (e.g., the volume difference between preferred variants).

On the other hand, the $\alpha / \alpha-\mathrm{OR}$ between variants within $\beta$ grain were examined by Wang et al. [20] and the fractions of 6 types of $\alpha / \alpha-\mathrm{OR}$ were calculated. They found that the variants of intragranular $\alpha$ precipitates are more likely to keep a $\alpha / \alpha-O R$ of $B, D$ or $F$ type with their neighbors according to statistic data from experimental images, which is consistent with our 
calculation. Using phenomenological theory of martensitic transformation, they attributed the formation of $\mathrm{B}$ and $\mathrm{D}$ types of $\alpha / \alpha-\mathrm{OR}$ to the accommodation of the shape strain of several variants in one cluster, which results in a smaller average strain and the accommodation of strain is usually happened among three or four variants of B and D type. However, the F type of $\alpha$ / $\alpha$-OR frequently observed experimentally between two adjacent variants $[3,17,20]$ is still lacking of satisfactory explanations [17]. This is likely due to the fact that the information of elastic interaction energy, the shape and relative orientation between variants are all missing in calculations based on phenomenological and/or mean-field approaches used by previous researchers. Through our current phase field simulation that incorporates detailed information on precipitate microstructure (including size, shape, orientation, and spatial distribution of precipitates), however, the F type (V1|V7) is also predicted and the formation of such $\alpha / \alpha-\mathrm{OR}$ is dominated by the elastic interaction during the nucleation.

\section{Conclusions}

In this paper the behavior of $\alpha$ precipitation near pre-existing $\alpha$ lath is investigated using a 3-D phase field model. The autocatalytic effect between primary and secondary $\alpha$ precipitates is observed and its influence on variant selection is analyzed. The major findings include the following:

1) The stress field around a pre-existing $\alpha$ lath is highly non-uniform, which will interact with the transformation strains of different $\alpha$ variants in distinct ways and therefore lead to the phenomenon of variant selection. The autocatalytic effects are attributed to the relaxation of local stress associated with pre-existing $\alpha$ lath and thus the reduction of elastic energy. Interaction energy analysis indicates that the relaxation of local stress field could be more efficiently achieved if the precipitated secondary $\alpha$ having: i) a highly negative interaction energy with the pre-existing $\alpha$ lath and ii) a habit plane orientation as close to that of the pre-existing $\alpha$ lath as possible. While this is confirmed in our 3-D phase field simulations, the mutual elastic interaction between spatially distributed pre-existing and secondary $\alpha$ precipitates predicts additional $\alpha$ variants that are missed in other mean-field analysis.

2) Based on the simulation results, the $\alpha / \alpha$-OR between each favored $\alpha$ variant and the pre-existing $\alpha$ is examined. The three most favored ones are found to belong to those most 
frequently observed types in experiments, i.e., $[11 \overline{2} 0]_{\alpha} / 60^{\circ}$-type, $[\overline{10} 553]_{\alpha} / 63.26^{\circ}$-type and $[0001]_{\alpha} / 10.53^{\circ}$-type.

3) A new mechanism for the formation of the typical basketweave structure in two-phase $\alpha / \beta$

Ti-alloys is proposed. Due to the elastic interaction between any two of the three variants forming the B type of $\alpha / \alpha-\mathrm{OR}$, the basketweave structure is likely to be formed through a "chain reaction" assisted by autocatalysis.

Therefore, the autocatalysis between $\alpha$ precipitates predicted in the current study may shed some light on the formation mechanism of $\alpha$ clusters consisting of $\alpha$ variants with specific $\alpha / \alpha-O R$.

\section{Acknowledgements}

This work is supported by the 973 Programs under Giants No. 2012CB619600 and No. 2014 CB644003 (D.Q and W.L), the US National Science Foundation DMREF program under Grant No. DMR-1435483 and the American Lightweight Metals Innovation Institute (P.Z., R.S and Y.W).

\section{Figure captions:}

Fig. 1 Growth of an $\alpha$ precipitate: (a) initial nucleus, (b)-(f) growth of the $\alpha$ precipitate into a plate with well-defiend habit plane of $(\overline{11} 1311)_{\beta}$.

Fig. 2 Stress fields around a semi-coherent $\alpha$ plate in the cross section perpendicular to $\mathrm{z} \|[101]_{\beta}$.

Fig. 3 The type, shape and distribution of variants induced by the primary $\alpha(\mathrm{V} 1)$ plate near its surface and edge. Different colors represent different $\alpha$ orientation variants.

Fig. 4 Calculation of interaction energy between pre-existing $\alpha$ lath and each variant: (a) the minimum interaction energy $E_{\min }^{\text {int }}(p)$ between each secondary variant and primary V1 lath, (b)-(l) distribution of most favorable nucleation sites for each variant in the presence of V1 based on interaction energy calculation.

Fig. 5 (a) 3-D morphology of an $\alpha$ cluster consisting of three variants sharing a common $[11 \overline{2} 0]_{\alpha}$ from phase field simulation, (b) 2-D morphology of cross section indicated in (a), (c) schematic drawing of auto-catalytic effects between variants of B type $\alpha / \alpha-\mathrm{OR}$ and its possible relationship with the development of basketweave structure.

Fig. 6 The habit plane inclination angles $\varphi_{h p}$ between primary V1 lath and variants forming 
different $\alpha / \alpha-\mathrm{OR}$ with $\mathrm{V} 1$.

\section{Reference}

[1] D. Bhattacharyya, G. Viswanathan, R. Denkenberger, D. Furrer, H.L. Fraser, The role of crystallographic and geometrical relationships between $\alpha$ and $\beta$ phases in an $\alpha / \beta$ titanium alloy, Acta Materialia, 51 (2003) 4679-4691.

[2] D. Banerjee, J. Williams, Perspectives on Titanium Science and Technology, Acta Materialia, 61 (2013) 844-879.

[3] S.M.C. van Bohemen, A. Kamp, R.H. Petrov, L.A.I. Kestens, J. Sietsma, Nucleation and variant selection of secondary $\alpha$ plates in a $\beta$ Ti alloy, Acta Materialia, 56 (2008) 5907-5914.

[4] S.L. Semiatin, K.T. Kinsel, A.L. Pilchak, G.A. Sargent, Effect of Process Variables on Transformation-Texture Development in Ti-6Al-4V Sheet Following Beta Heat Treatment, Metallurgical and Materials Transactions A, 44 (2013) 3852-3865.

[5] D. Bhattacharyya, G. Viswanathan, H.L. Fraser, Crystallographic and morphological relationships between $\beta$ phase and the Widmanstätten and allotriomorphic $\alpha$ phase at special $\beta$ grain boundaries in an $\alpha / \beta$ titanium alloy, Acta Materialia, 55 (2007) 6765-6778.

[6] K. Morii, H. Mecking, G. Lütjering, Y. Nakayama, Stability of the texture of Ti-6Al-4V during rolling in the two phase field, Scripta metallurgica, 20 (1986) 1795-1800.

[7] L. Germain, N. Gey, M. Humbert, P. Bocher, M. Jahazi, Analysis of sharp microtexture heterogeneities in a bimodal IMI 834 billet, Acta materialia, 53 (2005) 3535-3543.

[8] M. Humbert, L. Germain, N. Gey, P. Bocher, M. Jahazi, Study of the variant selection in sharp textured regions of bimodal IMI 834 billet, Materials Science and Engineering: A, 430 (2006) 157-164.

[9] J. Da Costa Teixeira, B. Appolaire, E. Aeby-Gautier, S. Denis, L. Héricher, Modeling of the phase transformations in near- $\beta$ titanium alloys during the cooling after forging, Computational Materials Science, 42 (2008) 266-280.

[10] D. Banerjee, J.C. Williams, Perspectives on Titanium Science and Technology, Acta Materialia, 61 (2013) 844-879.

[11] W. Burgers, On the process of transition of the cubic-body-centered modification into the hexagonal-close-packed modification of zirconium, Physica, 1 (1934) 561-586.

[12] R. Shi, N. Ma, Y. Wang, Predicting equilibrium shape of precipitates as function of coherency state, Acta Materialia, 60 (2012) 4172-4184.

[13] E. Lee, R. Banerjee, S. Kar, Selection of a variants during microstructural evolution in a/b titanium alloys, Philosophical Magazine, 87 (2007) 3615-3627.

[14] N. Gey, M. Humbert, M. Philippe, Y. Combres, Modeling the transformation texture of Ti-64 sheets after rolling in the $\beta$-field, Materials Science and Engineering: A, 230 (1997) 68-74.

[15] T. Furuhara, T. Maki, Variant selection in heterogeneous nucleation on defects in diffusional phase transformation and precipitation, Materials Science and Engineering: A, 312 (2001) 145-154.

[16] N. Gey, M. Humbert, Characterization of the variant selection occurring during the $\alpha \rightarrow \beta \rightarrow \alpha$ phase transformations of a cold rolled titanium sheet, Acta materialia, 50 (2002) 277-287.

[17] E. Lee, R. Banerjee, S. Kar, D. Bhattacharyya, H.L. Fraser, Selection of $\alpha$ variants during microstructural evolution in $\alpha / \beta$ titanium alloys, Philosophical Magazine, 87 (2007) 3615-3627.

[18] M. Salib, J. Teixeira, L. Germain, E. Lamielle, N. Gey, E. Aeby-Gautier, Influence of transformation temperature on microtexture formation associated with $\alpha$ precipitation at $\beta$ grain 
boundaries in a $\beta$ metastable titanium alloy, Acta Materialia, 61 (2013) 3758-3768.

[19] R. Shi, V. Dixit, H.L. Fraser, Y. Wang, Variant selection of grain boundary $\alpha$ by special prior $\beta$ grain boundaries in titanium alloys, Acta Materialia, 75 (2014) 156-166.

[20] S. Wang, M. Aindow, M. Starink, Effect of self-accommodation on $<\mathrm{i}>\alpha</ \mathrm{i}>/<\mathrm{i}>\alpha</ \mathrm{i}>$ boundary populations in pure titanium, Acta materialia, 51 (2003) 2485-2503.

[21] V. Dixit, Grain-Boundary Parameters Controlled Allotriomorphic Phase Transformations in Beta-Processed Titanium Alloys, in, The Ohio State University, 2013.

[22] S.V. Divinski, V.N. Dnieprenko, O.M. Ivasishin, Effect of phase transformation on texture formation in Ti-base alloys, Materials Science and Engineering: A, 243 (1998) 201-205.

[23] K. Madangopal, J.B. Singh, S. Banerjee, The nature of self-accommodation in Ni Ti shape memory alloys, Scripta metallurgica et materialia, 29 (1993) 725-728.

[24] A.G. Khachaturian, Theory of structural transformations in solids, 1983.

[25] D. Qiu, R. Shi, D. Zhang, W. Lu, Y. Wang, Variant selection by dislocations during $\alpha$ precipitation in $\alpha / \beta$ titanium alloys, Acta Materialia, 88 (2015) 218-231.

[26] J.D. Eshelby, The determination of the elastic field of an ellipsoidal inclusion, and related problems, Proceedings of the Royal Society of London. Series A. Mathematical and Physical Sciences, 241 (1957) 376-396.

[27] N. Moelans, B. Blanpain, P. Wollants, An introduction to phase-field modeling of microstructure evolution, Calphad, 32 (2008) 268-294.

[28] L.-Q. Chen, Phase-field models for microstructure evolution, Annual review of materials research, 32 (2002) 113-140.

[29] C. Shen, Y. Wang, Incorporation of $\gamma$-surface to phase field model of dislocations: simulating dislocation dissociation in fcc crystals, Acta materialia, 52 (2004) 683-691.

[30] Y. Wang, J. Li, Phase field modeling of defects and deformation, Acta Materialia, 58 (2010) 1212-1235.

[31] R. Shi, Y. Wang, Variant selection during $\alpha$ precipitation in Ti-6Al-4V under the influence of local stress - A simulation study, Acta Materialia, 61 (2013) 6006-6024.

[32] Y.-Z. Wang, N. Ma, Q. Chen, F. Zhang, S. Chen, Y. Chang, Predicting phase equilibrium, phase transformation, and microstructure evolution in titanium alloys, JOM, 57 (2005) 32-39.

[33] J.W. Cahn, J.E. Hilliard, Free Energy of a Nonuniform System. I. Interfacial Free Energy, The Journal of Chemical Physics, 28 (1958) 258.

[34] I. Steinbach, F. Pezzolla, A generalized field method for multiphase transformations using interface fields, Physica D: Nonlinear Phenomena, 134 (1999) 385-393.

[35] S. Gyoon Kim, W. Tae Kim, T. Suzuki, M. Ode, Phase-field modeling of eutectic solidification, Journal of Crystal Growth, 261 (2004) 135-158.

[36] W. Luo, C. Shen, Y. Wang, Nucleation of ordered particles at dislocations and formation of split patterns, Acta materialia, 55 (2007) 2579-2586.

[37] H. Liu, Y. Gao, Y.M. Zhu, Y. Wang, J.F. Nie, A simulation study of $\beta 1$ precipitation on dislocations in an Mg-rare earth alloy, Acta Materialia, 77 (2014) 133-15.

[38] H. Liu, Y. Gao, Z. Xu, Y.M. Zhu, Y. Wang, J.F. Nie, Guided Self-Assembly of Nano-Precipitates into Mesocrystals, Sci Rep, 5 (2015) 16530.

[39] D. Qiu, R. Shi, P. Zhao, D. Zhang, W. Lu, Y. Wang, Effect of low-angle grain boundaries on morphology and variant selection of grain boundary allotriomorphs and Widmanstätten side-plates. Acta Materialia 112 (2016): 347-360. 


\section{${ }^{\star}$ Graphical Abstracts (for review)}

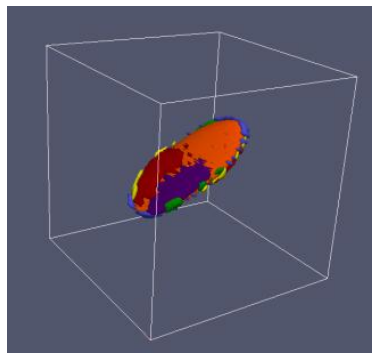

Elastic interaction

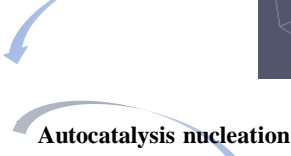

Autocatalysis nucleation

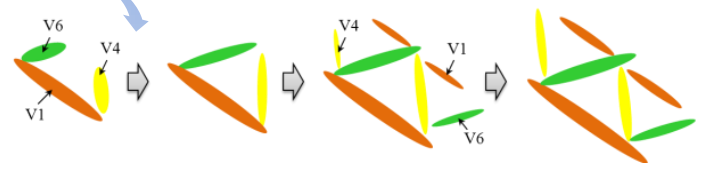




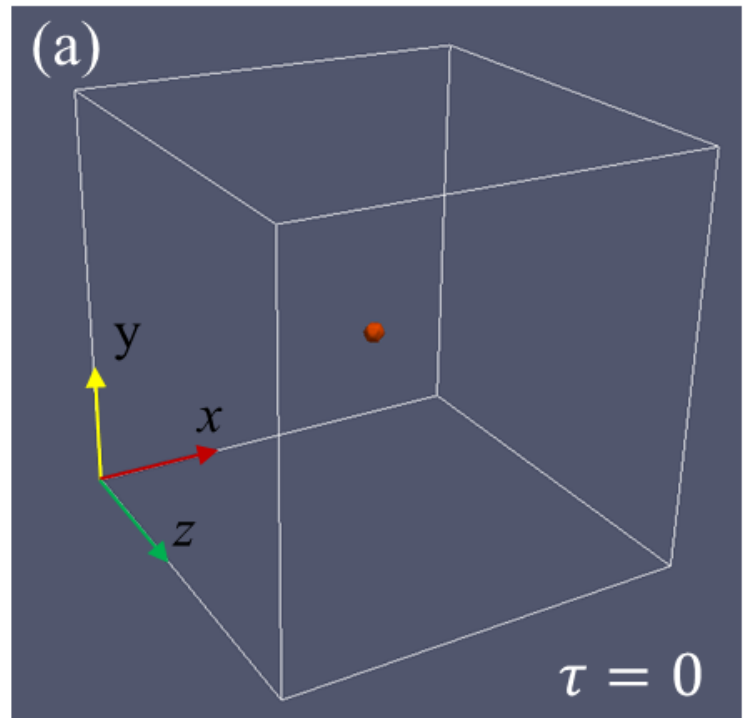

(b)

(d)

$$
\tau=5
$$

(e)
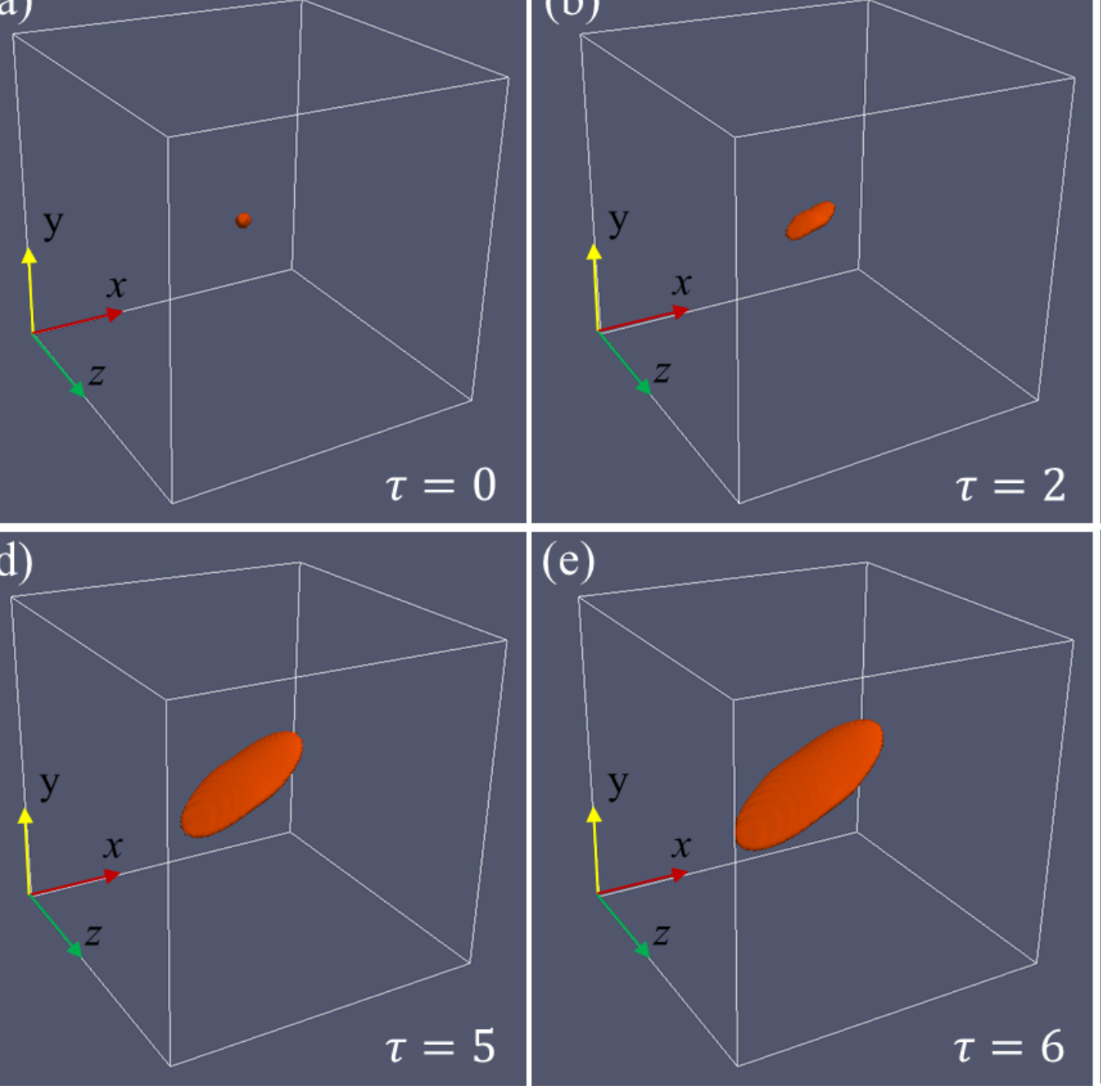

(c)

$$
\tau=4
$$

\section{(f)}

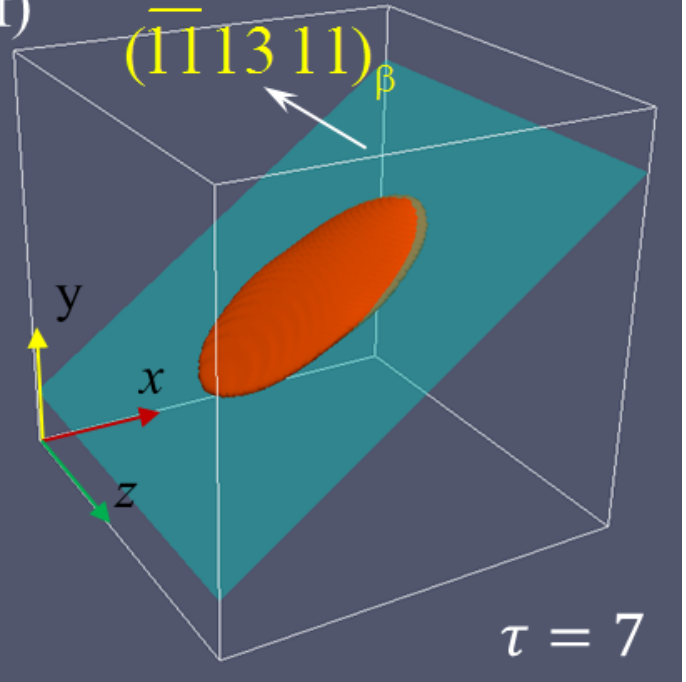

Fig. 1 Growth of an $\alpha$ precipitate: (a) initial nucleus, (b)-(f) growth of the $\alpha$ precipitate into a plate with well-defiend habit plane of $(\overline{11} 1311)_{\beta}$. 

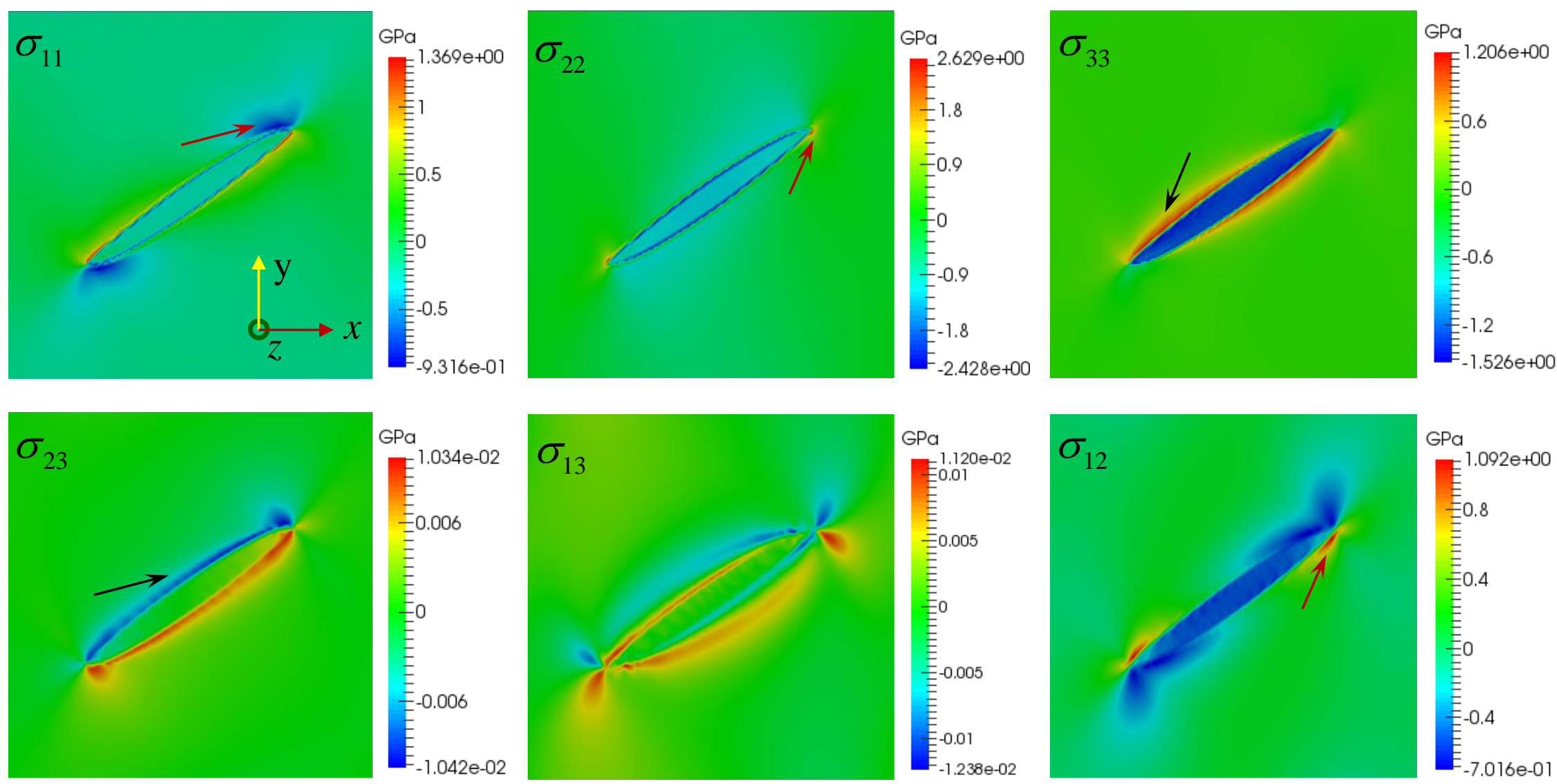

Fig. 2 Stress fields around a semi-coherent $\alpha$ plate in the cross section perpendicular to $\mathrm{z}||[101]_{\beta}$. 


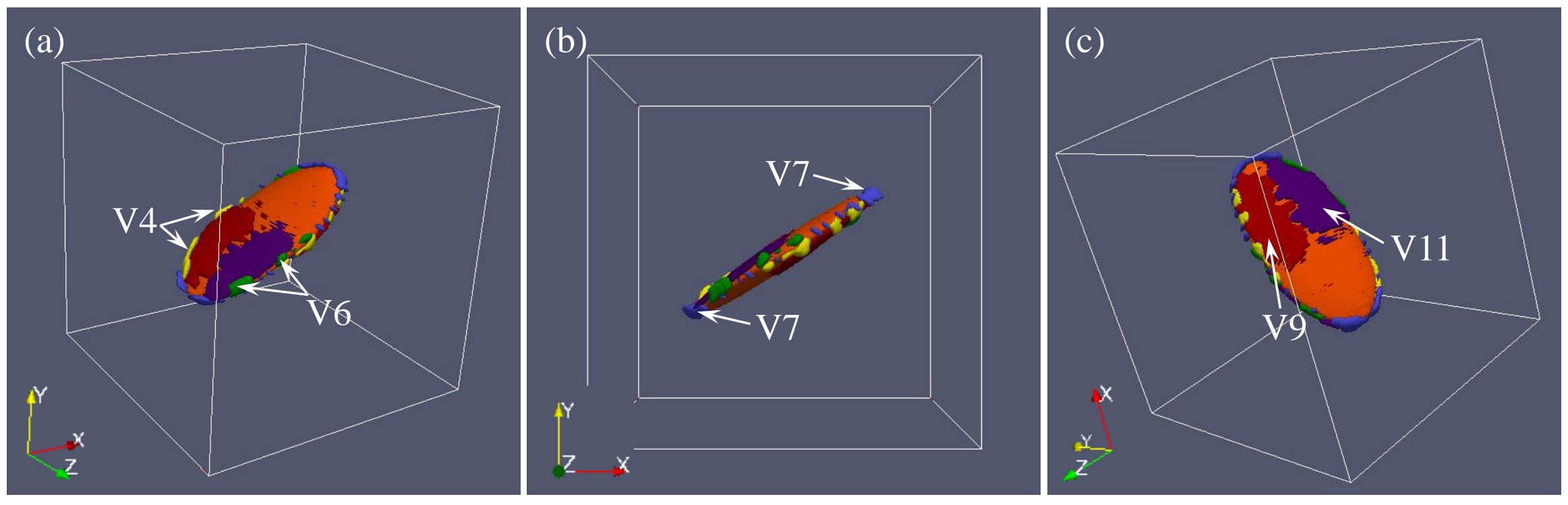

Fig. 3 The type, shape and distribution of variants induced by the primary $\alpha$ (V1) plate near its surface and edge. Different colors represent different $\alpha$ orientation variants. 


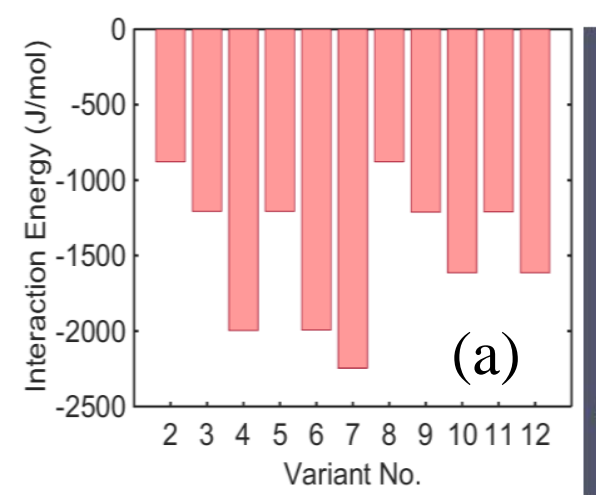

(b)

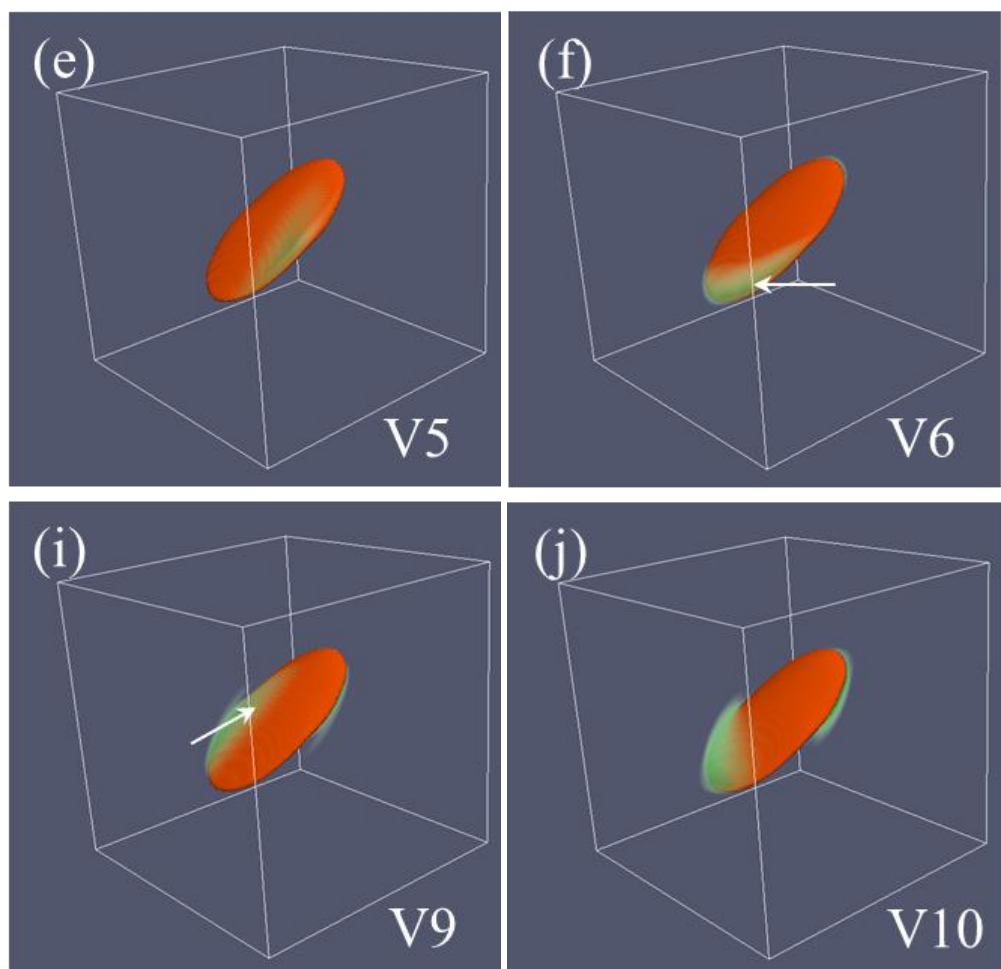

(c)

(d)

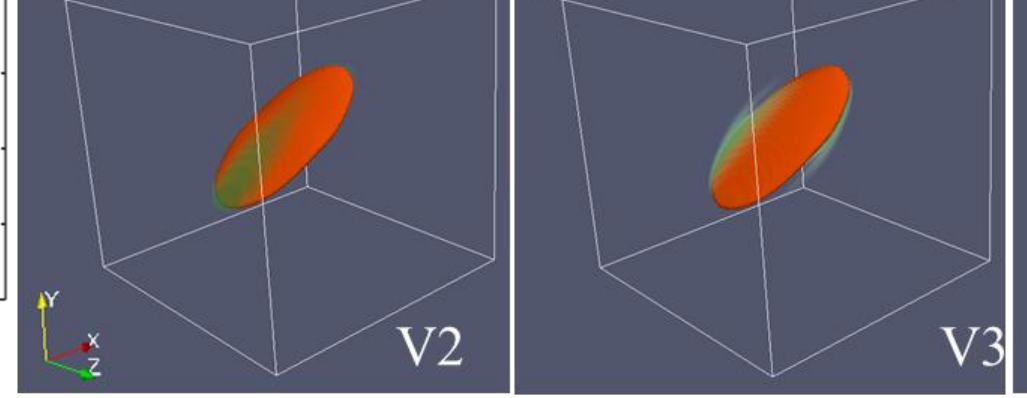

(g)

\section{(k)}

V11 (h)

V7

Fig. 4 Calculation of interaction energy between pre-existing $\alpha$ lath and each variant: (a) the minimum interaction energy $E_{\min }^{\mathrm{int}}(p)$ between each secondary variant and primary V1 lath, (b)-(l) distribution of most favorable nucleation sites for each variant in the presence of V1 based on interaction energy calculation. 

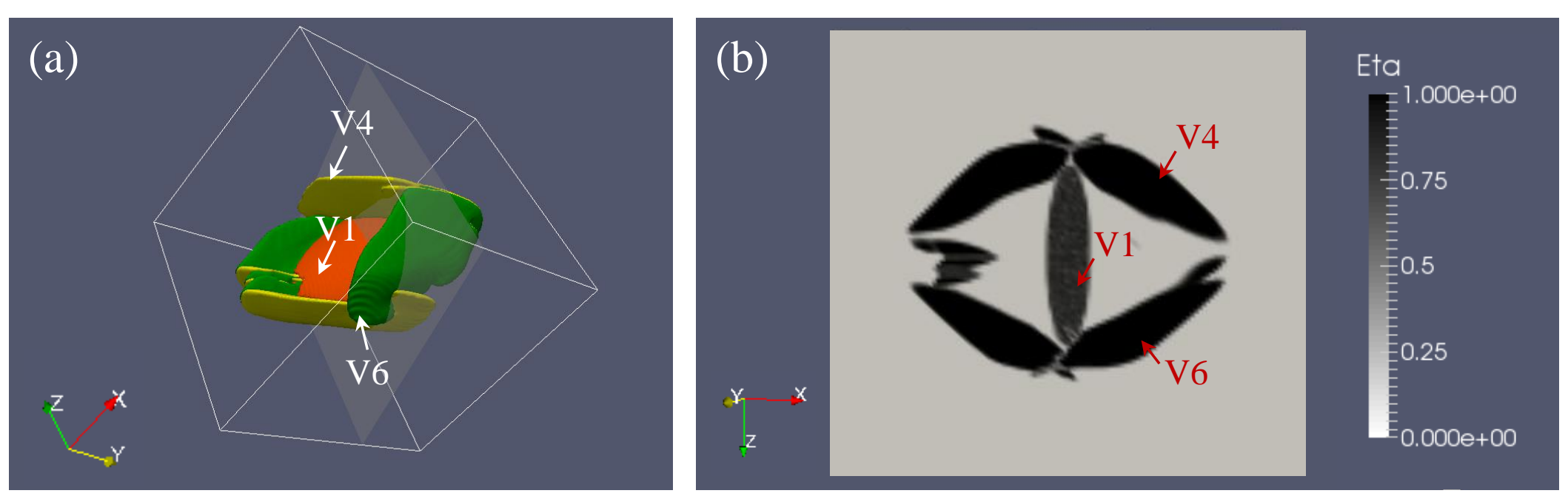

(c)
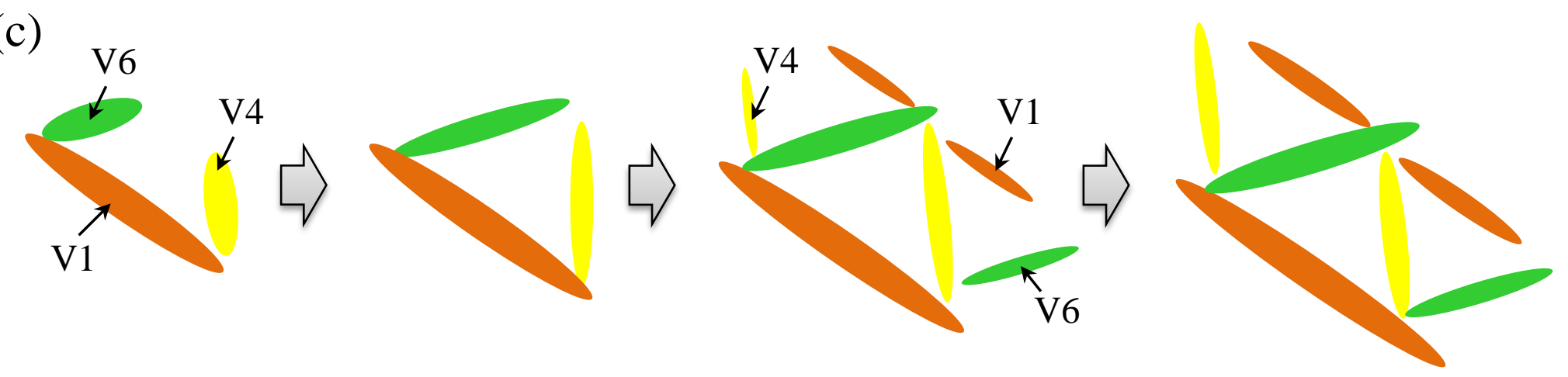

Fig. 5 (a) 3-D morphology of an $\alpha$ cluster consisting of three variants sharing a common $[11 \overline{2} 0]_{\alpha}$ from phase field simulation, (b) 2-D morphology of cross section indicated in (a), (c) schematic drawing of auto-catalytic effects between variants of B type $\alpha / \alpha-\mathrm{OR}$ and its possible relationship with the development of basketweave structure. 


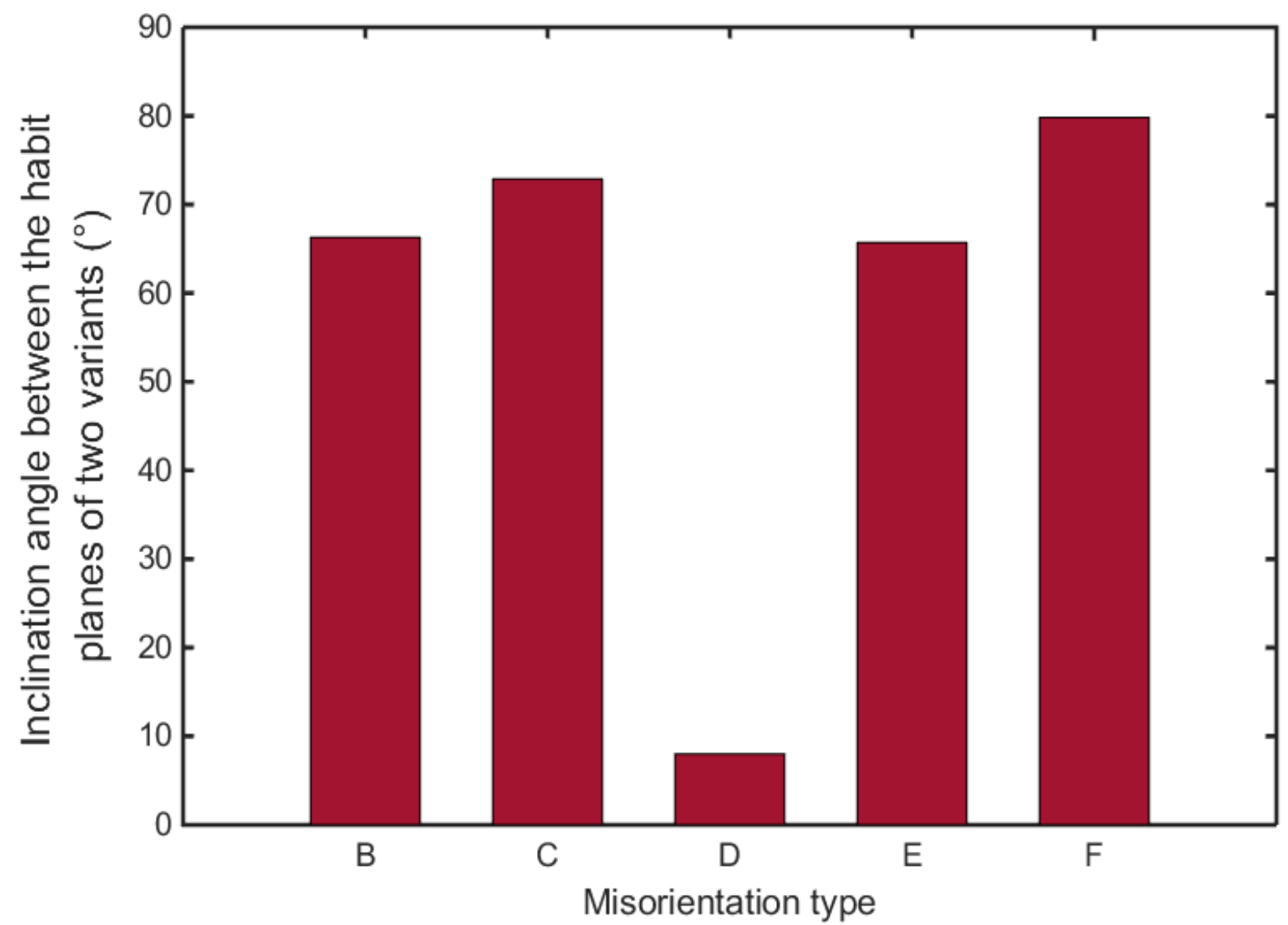

Fig. 6 The habit plane inclination angles $\varphi_{h p}$ between primary V1 lath and variants forming different $\alpha / \alpha-\mathrm{OR}$ with $\mathrm{V} 1$. 